\title{
Influence of thermal residual stresses on the composite macroscopic behavior
}

\author{
G.K. Hu ${ }^{\mathrm{a}, \mathrm{b}, *}$, G.J. Weng ${ }^{\mathrm{c}}$ \\ ${ }^{a}$ Department of Applied Mechanics, Beijing Institute of Technology, Beijing 100081, China \\ ${ }^{\mathrm{b}}$ LNM Institute of Mechanics, Academia Sinica, Beijing 100080, China \\ ${ }^{c}$ Department of Mechanical and Aerospace Engineering, Rutgers University, New Brunswick, NJ 08903, USA
}

Received 4 June 1997

\begin{abstract}
The influence of the thermal residual stress on the deformation behavior of a composite has been analyzed with a new micromechanical method. The method is based on secant moduli approximation and a new homogenized effective stress to characterize the plastic state of the matrix. It is found that the generated thermal residual stresses after cooling and their influence on the subsequent deformation behavior depends significantly on the aspect ratio of the inclusions. With prolate inclusions, the presence of thermal residual stresses generate a higher compressive hardening curves of the composite, but it is reversed with oblate inclusions. For particle reinforced composite, thermal residual stresses induce a tensile hardening curve higher than the compressive one and this is in agreement with experimental observations. (C) 1998 Elsevier Science Ltd.
\end{abstract}

Keywords: Metal matrix composite; Residual stress; Micromechanics; Second order stress moment; Plastic strain

\section{Introduction}

Due to a mismatch in the coefficients of thermal expansion between the matrix and inclusions, change in temperature produces an internal stress in the composite. These thermal stresses may be partially relaxed by the plastic deformation of the ductile matrix and lead to additional hardening of the matrix in the composite (Taya and Mori, 1987; Shibata et al., 1992; Ramakrishnan, 1996). Since the thermal effects are induced before mechanical testing, their presence can induce the asymmetry in the tensile and compressive yield stresses of the composite (Arsenault and Taya, 1987; Corbin et al., 1991).

Because of its influence on the properties, the residual stress in composites has been the subject of several studies, both experimentally and analytically. The equivalent inclusion method (Eshelby, 1957) is usually used to evaluate thermal residual stresses of a composite (Takao and Taya, 1985; Withers et al., 1989). The average state of the internal stresses in the matrix can be readily calculated. A simple way to analyze the plastic behavior

\footnotetext{
* Corresponding author. 
of a composite is to define the effective stress of the matrix directly by $\sigma_{\text {eff }}^{2}=3 / 2\langle s\rangle_{0}:\langle s\rangle_{0}$, where $s$ is the deviatoric part of local stress $\boldsymbol{\sigma}$ and $\langle\cdot\rangle_{0}$ means the volume average of the said quantity over the matrix phase. This approach is usually referred to as the mean stress approach (Qiu and Weng, 1992). However, for an isotropic composite, the temperature change induces an average stress in a hydrostatic form in the matrix, so such a defined effective stress of the matrix fails to predict the plastic flow of the composite.

To overcome this difficulty, Qiu and Weng (1992) defined an homogenized effective stress of the matrix directly from the average of the local effective stress, that is $\sigma_{\text {eff }}^{2}=3 / 2\langle s: s\rangle_{0}$, and this homogenized stress is believed to account to some extent for the influence of the local stress fluctuations in the matrix. Recently, Hu (1996) proposed a field-fluctuation method to evaluate this effective stress, and showed that the prediction of the nonlinear properties for a composite with the homogenized effective stress and the secant moduli method (Berveiller and Zaoui, 1979; Tandon and Weng, 1988) corresponds to Ponte Castaneda's lower bound approach (Ponte Castaneda, 1991). A similar approach was applied to analyze thermal stress relief due to plastic flow by Pan and Weng (1993), and to analyze the influence of residual stresses on the flow behavior for a stable dual-phase steel by Bhattacharyya et al. (1993). However, in their analyses the homogenized effective stress was only evaluated in an approximate manner. In this paper, a method will be proposed to calculate the homogenized matrix effective stress in the presence of thermal residual stresses and afterwards it will be used together with the secant moduli method to analyze the influence of residual stresses on the subsequent deformation behavior of a composite.

\section{Theoretical analysis}

\subsection{Description of problem}

The representative volume element (RVE) of the considered composite is assumed to consist of well aligned spheroidal inclusions and an isotropic ductile matrix. The elastic stiffness (compliance) tensors of the matrix and the inclusion are denoted by $\boldsymbol{L}_{0}$ and $\boldsymbol{L}_{1}\left(\boldsymbol{M}_{0}\right.$ and $\left.\boldsymbol{M}_{1}\right)$ respectively, and their thermal expansion coefficients by $\alpha_{0}$ and $\alpha_{1}$. The volume fraction of the inclusion is represented by $f$. The composite is subjected to a uniform temperature change, $\Delta T$, and after that a macroscopic stress $\boldsymbol{\Sigma}$ (or macroscopic strain $\boldsymbol{E}$ ) is applied. The temperature drop is assumed to be sufficiently fast so that the extent of plastic deformation in the matrix can be evaluated with the properties at the service temperature. For simplicity, the inclusions will be taken to be elastic.

The elastic case is first considered. The effect of temperature drop is simulated by an eigenstrain $\boldsymbol{\varepsilon}^{*}=\left(\alpha_{1}-\alpha_{0}\right) \boldsymbol{I} \Delta T$ inside of each inclusion. Now we will analyze the following situation: a composite consists of aligned spheroidal inclusions and, in each inclusion, there is an eigenstrain $\boldsymbol{\varepsilon}^{*}$ and along the boundary of RVE, a macroscopic stress $\boldsymbol{\Sigma}$ (or strain $\boldsymbol{E}$ ) is applied. The local stress $\boldsymbol{\sigma}$ inside the composite can be decomposed into two parts: $\boldsymbol{\sigma}^{\mathrm{I}}$, the stress owing to the applied load $\boldsymbol{\Sigma}$, and $\boldsymbol{\sigma}^{\mathrm{II}}$, the stress due to the eigenstrain $\boldsymbol{\varepsilon}^{*}$, so that $\boldsymbol{\sigma}=\boldsymbol{\sigma}^{\mathrm{I}}+\boldsymbol{\sigma}^{\mathrm{II}}$. The plastic flow of the composite is controlled by the detail local stresses in the matrix. However, it is difficult to perform an exact analysis on such local stresses through an analytical method. In this paper an approximate method will be proposed, in which an homogenized effective stress of the matrix $\left\langle\sigma_{\mathrm{e}}^{2}\right\rangle_{0}$ will be used to characterize the plastic state of the matrix in the composite, noting that $\sigma_{\mathrm{e}}$ is the classical von Mises' stress.

\subsection{Determination of the homogenized effective stress}

With the help of Eshelby's inclusion theory (Eshelby, 1957) and Mori-Tanaka's mean stress approach (Mori and Tanaka, 1973), we readily get (see more details in Appendix A) the compliance tensor of the composite $\boldsymbol{M}_{\mathrm{c}}$, 
and the average stress of the matrix $\langle\boldsymbol{\sigma}\rangle_{0}$ :

$$
\begin{aligned}
& \boldsymbol{M}_{\mathrm{c}}=\boldsymbol{M}_{0}+f\left\{\left[\boldsymbol{M}_{1} \boldsymbol{M}_{0}^{-1}-\boldsymbol{I}\right]^{-1}+(1-f)(\boldsymbol{I}-\boldsymbol{S})\right\}^{-1} \boldsymbol{M}_{0}, \\
& \langle\boldsymbol{\sigma}\rangle_{0}=\frac{1}{1-f}\left(\boldsymbol{M}_{1}-\boldsymbol{M}_{0}\right)^{-1}\left(\boldsymbol{M}_{1}-\boldsymbol{M}_{\mathrm{c}}\right) \boldsymbol{\Sigma}-\left\{\left(\boldsymbol{M}_{1}-\boldsymbol{M}_{0}\right)^{-1}\left(\boldsymbol{M}_{1}-\boldsymbol{M}_{\mathrm{c}}\right)-\boldsymbol{I}\right\} \boldsymbol{M}_{0}^{-1}(\boldsymbol{I}-\boldsymbol{S}) \boldsymbol{\varepsilon}^{*},
\end{aligned}
$$

where $\boldsymbol{I}$ is the unit tensor and $\boldsymbol{S}$ is the Eshelby tensor. Now following the method proposed by Bobeth and Diener (1987) and Kreher (1990) for an elastic composite (subjected to $\Delta T$ and $\boldsymbol{\Sigma}$ (or $\boldsymbol{E}$ )), the total strain energy of the composite is

$\left.U=\frac{1}{2}\left\langle\left(\boldsymbol{\sigma}^{\mathrm{I}}+\boldsymbol{\sigma}^{\mathrm{II}}\right): \boldsymbol{\varepsilon}^{\mathrm{I}}+\boldsymbol{\varepsilon}^{\mathrm{II}}-\boldsymbol{\varepsilon}^{*}\right)\right\rangle$.

It can be further written by using Hill's condition (Hill, 1963) as

$U=\frac{1}{2}\left\langle\left(\boldsymbol{\sigma}^{\mathrm{I}}: \boldsymbol{\varepsilon}^{\mathrm{I}}\right)\right\rangle-\frac{1}{2}\left\langle\left(\boldsymbol{\sigma}^{\mathrm{II}}: \boldsymbol{\varepsilon}^{*}\right\rangle=\frac{1}{2} \boldsymbol{\Sigma}: \boldsymbol{M}_{\mathrm{c}}: \boldsymbol{\Sigma}-\frac{1}{2}\left\langle\boldsymbol{\sigma}^{\mathrm{II}}: \boldsymbol{\varepsilon}^{*}\right\rangle\right.$.

The total elastic energy of the composite can also be written in the following form:

$U=\frac{1}{2}\left\langle\left(\boldsymbol{\sigma}^{\mathrm{I}}+\boldsymbol{\sigma}^{\mathrm{II}}\right): \boldsymbol{m}:\left(\boldsymbol{\sigma}^{\mathrm{I}}+\boldsymbol{\sigma}^{\mathrm{II}}\right)\right\rangle$.

where $\boldsymbol{m}$ is the local compliance tensor and $\langle\cdot\rangle$ means the average of the said quantity over the RVE. Keeping $\boldsymbol{\Sigma}$ and $\Delta T$ unchanged, while allowing the local compliance tensor $\boldsymbol{m}$ to have a variation $\delta \boldsymbol{m}$, this will induce the variations of the local stress and consequently the total strain energy of the composite

$$
\begin{aligned}
\delta U & =\frac{1}{2}\left\langle\left(\boldsymbol{\sigma}^{\mathrm{I}}+\boldsymbol{\sigma}^{\mathrm{II}}\right): \delta \boldsymbol{m}:\left(\boldsymbol{\sigma}^{\mathrm{I}}+\boldsymbol{\sigma}^{\mathrm{II}}\right)\right\rangle+\left\langle\left(\boldsymbol{\sigma}^{\mathrm{I}}+\boldsymbol{\sigma}^{\mathrm{II}}\right): \boldsymbol{m}: \delta\left(\boldsymbol{\sigma}^{\mathrm{I}}+\boldsymbol{\sigma}^{\mathrm{II}}\right)\right\rangle \\
& =\frac{1}{2}\left\langle\left(\boldsymbol{\sigma}^{\mathrm{I}}+\boldsymbol{\sigma}^{\mathrm{II}}\right): \delta \boldsymbol{m}:\left(\boldsymbol{\sigma}^{\mathrm{I}}+\boldsymbol{\sigma}^{\mathrm{II}}\right)\right\rangle-\left\langle\boldsymbol{\varepsilon}^{*}: \delta \boldsymbol{\sigma}^{\mathrm{I}}\right\rangle-\left\langle\boldsymbol{\varepsilon}^{*}: \delta \boldsymbol{\sigma}^{\mathrm{II}}\right\rangle .
\end{aligned}
$$

In the derivation of Eq. (6), Hill's condition and $\left\langle\delta\left(\boldsymbol{\sigma}^{\mathrm{I}}+\boldsymbol{\sigma}^{\mathrm{II}}\right)\right\rangle=0$ were used.

From Eq. (4) we get

$\delta U=\frac{1}{2} \boldsymbol{\Sigma}: \delta \boldsymbol{M}_{\mathrm{c}}: \boldsymbol{\Sigma}-\frac{1}{2}\left\langle\boldsymbol{\varepsilon}^{*}: \delta \boldsymbol{\sigma}^{\mathrm{II}}\right\rangle$

and thus

$$
\frac{1}{2}\left\langle\left(\boldsymbol{\sigma}^{\mathrm{I}}+\boldsymbol{\sigma}^{\mathrm{II}}\right): \delta \boldsymbol{m}:\left(\boldsymbol{\sigma}^{\mathrm{I}}+\boldsymbol{\sigma}^{\mathrm{II}}\right)\right\rangle=\frac{1}{2} \boldsymbol{\Sigma}: \delta \boldsymbol{M}_{\mathrm{c}}: \boldsymbol{\Sigma}+\left\langle\boldsymbol{\varepsilon}^{*}: \delta \boldsymbol{\sigma}^{\mathrm{I}}\right\rangle+\frac{1}{2}\left\langle\boldsymbol{\varepsilon}^{*}: \delta \boldsymbol{\sigma}^{\mathrm{II}}\right\rangle .
$$

In order to find $\boldsymbol{\sigma}_{\mathrm{e}}$ of the matrix, let only the shear modulus of the matrix have a variation $\delta \mu_{0}$. Then using the fact that $\varepsilon^{*}$ is only prescribed in the inclusions, Eq. (7) this time becomes

$(1-f) \delta\left(\frac{1}{2 \mu_{0}}\right) \frac{1}{2}\left\langle\left(\boldsymbol{s}^{\mathrm{I}}+\boldsymbol{s}^{\mathrm{II}}\right):\left(\boldsymbol{s}^{\mathrm{I}}+\boldsymbol{s}^{\mathrm{II}}\right)\right\rangle_{0}=\frac{1}{2} \boldsymbol{\Sigma}: \delta \boldsymbol{M}_{\mathrm{c}}: \boldsymbol{\Sigma}+f \boldsymbol{\varepsilon}^{*}:\left\langle\delta \boldsymbol{\sigma}^{\mathrm{I}}\right\rangle_{1}+\frac{1}{2} f \boldsymbol{\varepsilon}^{*}:\left\langle\delta \boldsymbol{\sigma}^{\mathrm{II}}\right\rangle_{1}$.

$\langle\cdot\rangle_{1}$ denotes the average of the said quantity over the inclusions. With the aid of the results given in Appendix A, $\left\langle\boldsymbol{\sigma}^{\mathrm{I}}\right\rangle_{1}=\boldsymbol{Q} \boldsymbol{\Sigma},\left\langle\boldsymbol{\sigma}^{\mathrm{II}}\right\rangle_{1}=\boldsymbol{R} \boldsymbol{\varepsilon}^{*}$, we get

$$
\begin{aligned}
\sigma_{\text {eff }}^{2}=\frac{3}{2}\left\langle\left(\boldsymbol{s}^{\mathrm{I}}+\boldsymbol{s}^{\mathrm{II}}\right):\left(\boldsymbol{s}^{\mathrm{I}}+\boldsymbol{s}^{\mathrm{II}}\right)\right\rangle_{0}= & \boldsymbol{\Sigma}:\left(-\frac{3 \mu_{0}^{2}}{1-f} \frac{\delta \boldsymbol{M}_{\mathrm{c}}}{\delta \mu_{0}}\right): \boldsymbol{\Sigma}+\boldsymbol{\varepsilon}^{*}:\left(-\frac{6 f \mu_{0}^{2}}{1-f} \frac{\delta \boldsymbol{Q}}{\delta \mu_{0}}\right): \boldsymbol{\Sigma} \\
& +\boldsymbol{\varepsilon}^{*}:\left(-\frac{3 f \mu_{0}^{2}}{1-f} \frac{\delta \boldsymbol{R}}{\delta \mu_{0}}\right): \boldsymbol{\varepsilon}^{*} .
\end{aligned}
$$


It is seen that the presence of residual stresses alters the yield properties of the composite through the last two terms of Eq. (8).

It is of interest to examine in detail a spherical inclusion reinforced composite, which represents a fundamental microgeometry of the two phase system. In this case $\varepsilon^{*}$ has a hydrostatic form, so the last two terms of Eq. (8) can be further simplified into a product of two hydrostatic terms, that is $\boldsymbol{\varepsilon}^{*}:\left\langle\boldsymbol{\sigma}^{\mathrm{I}}\right\rangle_{1}=1 / 3 \operatorname{tr} \boldsymbol{\varepsilon}^{*}$ $\left\langle\operatorname{tr} \boldsymbol{\sigma}^{\mathrm{I}}\right\rangle_{1}$ and $\operatorname{tr} \mathbf{A}=A_{i i}=A_{11}+A_{22}+A_{33}$. From Appendix A, we have

$\left\langle\operatorname{tr} \boldsymbol{\sigma}^{\mathrm{I}}\right\rangle_{1}=\frac{k_{1}}{(1-f)(\alpha-1)\left(k_{1}-k_{0}\right)+k_{1}} \operatorname{tr} \boldsymbol{\Sigma}, \quad\left\langle\operatorname{tr} \boldsymbol{\sigma}^{\mathrm{II}}\right\rangle_{1}=\frac{3 k_{1} k_{0}(1-f)(\alpha-1)}{(1-f)(\alpha-1)\left(k_{1}-k_{0}\right)+k_{1}} \operatorname{tr} \boldsymbol{\varepsilon}^{*}$.

Together with $\alpha=\left(1+\nu_{0}\right) / 3\left(1-\nu_{0}\right), \beta=2\left(4-5 \nu_{0}\right) / 15\left(1-\nu_{0}\right)$, Eq. (8) leads to

$\sigma_{\text {eff }}^{2}=\frac{\Sigma_{\mathrm{e}}^{2}}{A^{2}}+\frac{(\operatorname{tr} \boldsymbol{\Sigma})^{2}}{B^{2}}-C(\operatorname{tr} \boldsymbol{\Sigma})+D$

where

$$
\begin{aligned}
A^{2} & =\frac{\left[(1-f)(\beta-1)\left(\mu_{1}-\mu_{0}\right)+\mu_{1}\right]^{2}}{\left(\mu_{1}-\mu_{0}\right)^{2}\left[(1-f)\left(\beta^{2}-\beta\right)-1 / 5 f \alpha(1-\alpha)\right]+\left(\mu_{1}^{2}-\mu_{0}^{2}\right) \beta+\mu_{0}^{2}}, \\
B^{2} & =\frac{4\left[(1-f)(\alpha-1)\left(k_{1}-k_{0}\right)+k_{1}\right]^{2}}{f(1-\alpha)^{2}\left(k_{1}-k_{0}\right)^{2}}, \quad C=\frac{24 f k_{0} k_{1}\left(k_{1}-k_{0}\right)}{\left(3 k_{0} k_{1} / \mu_{0}+4 k_{0}-4 f k_{0}+4 f k_{1}\right)^{2}} \operatorname{tr} \boldsymbol{\varepsilon}^{*}, \\
D & =\frac{36 f k_{0}^{2} k_{1}^{2}}{\left(3 k_{0} k_{1} / \mu_{0}+4 k_{0}-4 f k_{0}-4 f k_{1}\right)^{2}}\left(\operatorname{tr} \boldsymbol{\varepsilon}^{*}\right)^{2}
\end{aligned}
$$

and $\Sigma_{\mathrm{e}}$ is the macroscopic von Mises' stress related to $\boldsymbol{\Sigma}$. It is seen that for a particle-reinforced composite, the presence of the residual stress influences indeed the composite yield properties through the constants $C$ and $D$. If the effective stress of the matrix is calculated directly from the matrix average stress $\sigma_{\text {eff }}^{2}=3 / 2\left\langle s^{I}+\right.$ $\left.\left.\left.\boldsymbol{s}^{\mathrm{II}}\right)\right\rangle_{0}:\left(\boldsymbol{s}^{\mathrm{I}}+\boldsymbol{s}^{\mathrm{II}}\right)\right\rangle_{0}$, the variation of the temperature in the absence of the applied stress would yield $\left\langle\boldsymbol{s}^{\mathrm{II}}\right\rangle_{0}=0$, so $\sigma_{\text {eff }}^{2}=3 / 2\left\langle s^{\mathrm{I}}\right\rangle:\left\langle\boldsymbol{s}^{\mathrm{I}}\right\rangle_{0}=0$. This would mean residual stresses have no influence on the plastic flow of the composite. With the new homogenized effective stress, this difficulty is removed.

\section{Nonlinear behavior of the composite in the presence of residual stress}

\subsection{Plastic flow due to thermal stress}

In the absence of the applied stress $\Sigma=0$, the onset of yielding of the composite owing to thermal stress can be determined by checking if the condition $\sigma_{\text {eff }}=\sigma_{\mathrm{y}}$ is satisfied ( $\sigma_{\mathrm{y}}$ the initial yield stress of the matrix). When the thermal stresses are large enough, the plastic flow will be generated in the matrix. To account for the change of the matrix constraint power on the inclusions during the plastic flow, as Berveiller and Zaoui (1979) did for the self-consistent method for polycrystal plasticity and Tandon and Weng (1988) for Mori-Tanaka's mean field theory, the secant moduli concept will be utilized. The matrix stress-plastic strain is taken to be of a power law type

$\sigma_{\mathrm{e}}=\sigma_{\mathrm{y}}+h\left(\varepsilon_{\mathrm{e}}^{\mathrm{p}}\right)^{n}$

where $\sigma_{\mathrm{e}}, \varepsilon_{\mathrm{e}}^{\mathrm{p}}$ are von Mises' effective stress and effective plastic strain respectively. The matrix secant shear and bulk moduli are defined by:

$\mu_{0}^{\mathrm{s}}=1 /\left(1 / \mu_{0}+3 \varepsilon_{\mathrm{e}}^{\mathrm{p}} /\left(\sigma_{\mathrm{y}}+h\left(\varepsilon_{\mathrm{e}}^{\mathrm{p}}\right)^{n}\right)\right), \quad k_{0}^{\mathrm{s}}=k_{0}$. 
The corresponding secant stiffness tensor of the matrix is denoted by $\boldsymbol{L}_{0}^{\mathrm{s}}$ (which is isotropic). Now the induced residual stresses at the end of cooling will be analyzed by the secant moduli method. Taking a linearized composite as the comparison composite with a matrix stiffness tensor set equal to the matrix secant stiffness tensor of the studied composite at the end of the cooling, the homogenized effective stress of the matrix for the linearized composite can be written as:

$\sigma_{\text {eff }}^{2}=\boldsymbol{\varepsilon}^{*}:\left(-\frac{3 f \mu_{0}^{2}}{1-f} \frac{\delta \boldsymbol{R}^{\mathrm{s}}}{\delta \mu_{0}}\right): \boldsymbol{\varepsilon}^{*}=\left[\sigma_{\mathrm{y}}+h\left(\varepsilon_{\mathrm{e}}^{\mathrm{p}}\right)^{n}\right]^{2}$,

where $\boldsymbol{R}^{\mathrm{s}}$ has the same expression as $\boldsymbol{R}$ with the condition that $\boldsymbol{L}_{0}$ be replaced by $\boldsymbol{L}_{0}^{\mathrm{s}}$. Henceforth the superscript $\mathrm{s}$ refers to the secant quantity which has the same form of expression as its elastic counterpart with this condition. The Eshelby tensor $\boldsymbol{S}^{\mathrm{s}}$ in this case depends on the secant Poisson's ratio of the matrix. Eq. (12) allows one to derive the homogenized effective plastic strain of the matrix $\varepsilon_{\mathrm{e}}^{\mathrm{p} 0}$ at the end of cooling. The induced average stresses in the matrix are determined by:

$\langle\sigma\rangle_{0}^{\mathrm{T}}=\left(\boldsymbol{M}_{1}-\boldsymbol{M}_{0}^{\mathrm{s}}\right)^{-1}\left(\boldsymbol{M}_{\mathrm{c}}^{\mathrm{s}}-\boldsymbol{M}_{0}^{\mathrm{s}}\right) \boldsymbol{M}_{0}^{\mathrm{s}-1}\left(\boldsymbol{I}-\boldsymbol{S}^{\mathrm{s}}\right) \varepsilon^{*}$,

where $\boldsymbol{M}_{0}^{\mathrm{s}}$ is the inverse of $\boldsymbol{L}_{0}^{\mathrm{s}}$ and $\boldsymbol{M}_{\mathrm{c}}^{\mathrm{s}}$ is the secant compliance tensor of the composite.

\subsection{Subsequent plastic deformation due to the applied stress $\mathbf{\Sigma}$}

After cooling, an internal stress is stocked in the matrix and its average value is given by Eq. (13). Besides this, the initial yield stress of the matrix written in the form of effective stress now becomes $\sigma_{\mathrm{y}}+h\left(\varepsilon_{\mathrm{e}}^{\mathrm{p} 0}\right)^{n}$. In short, the effects induced by the temperature drop before mechanical testing on the subsequent deformation of the composite are: (a) the yield stress of the matrix is enhanced and (b) the internal stresses in the inclusion and the matrix are generated. If there were not such internal stresses, the composite would have had a linear region determined by the elastic properties of the inclusion and matrix.

Depending on the nature of macroscopic applied stresses, these internal stresses can promote or inhibit further plastic flow of the composite. To account for the influence of the internal stresses on subsequent plastic flow of the composite, we will define a prescribed strain $\varepsilon^{\mathrm{H}}$ in the inclusion for the elastic composite to simulate the internal stresses at the end of cooling. Then we will consider the following problem: the composite is subjected to a macroscopic stress $\boldsymbol{\Sigma}$, in each inclusion there is an eigenstrain $\boldsymbol{\varepsilon}^{\mathrm{H}}$ and the matrix hardening law is given by $\sigma_{\mathrm{e}}=\sigma_{\mathrm{y}}+h\left(\varepsilon_{\mathrm{e}}^{\mathrm{p} 0}+\varepsilon_{\mathrm{e}}^{\mathrm{p}}\right)^{n}$. The plastic deformation of the matrix induced by the macroscopic applied stress is taken into account by $\varepsilon_{\mathrm{e}}^{\mathrm{p}}$. Again with the secant moduli method and the previously defined homogenized effective stress of the matrix, the nonlinear stress and strain relation of the composite can be derived. The method for doing this can be described as: for a composite subjected to an applied load $\boldsymbol{\Sigma}$, if the corresponding homogenized effective plastic strain of the matrix $\varepsilon_{\mathrm{e}}^{\mathrm{p}}$ is known (thus the matrix secant stiffness $\boldsymbol{L}_{0}^{\mathrm{s}}$ ), the secant compliance tensor $\boldsymbol{M}_{\mathrm{c}}^{\mathrm{s}}$ of the nonlinear composite is taken to be equal to the elastic compliance tensor of a linear comparison composite, and the matrix of the linear comparison composite has the elastic stiffness tensor $\boldsymbol{L}_{0}^{\mathrm{s}}$. The nonlinear composite strain $\boldsymbol{E}$ is related to the applied stress $\boldsymbol{\Sigma}$ simply by $\boldsymbol{E}=\boldsymbol{M}_{\mathrm{c}}^{\mathrm{s}} \boldsymbol{\Sigma}$. It is seen that to derive the stress and strain relation of a nonlinear composite, one has to provide a method to determine the relation between $\boldsymbol{\Sigma}$ and $\varepsilon_{\mathrm{e}}^{\mathrm{p}}$. The way proposed in this paper is to set the previously defined homogenized effective stress (Eq. (8)) into the matrix hardening law, and this provides a relationship to determine for a given $\boldsymbol{\Sigma}$ the corresponding $\varepsilon_{\mathrm{e}}^{\mathrm{p}}$ and then the stress-strain relation of the nonlinear composite can be successively evaluated.

\section{Numerical applications}

The proposed method is applied to a SiC/Al composite analyzed by Corbin et al. $(1991,1996)$ and Corbin and Wilkinson (1994a,b). The material constants for the matrix are: $E_{0}=73 \mathrm{GPa}, \nu_{0}=0.33, \sigma_{\mathrm{y}}=160 \mathrm{MPa}$, 


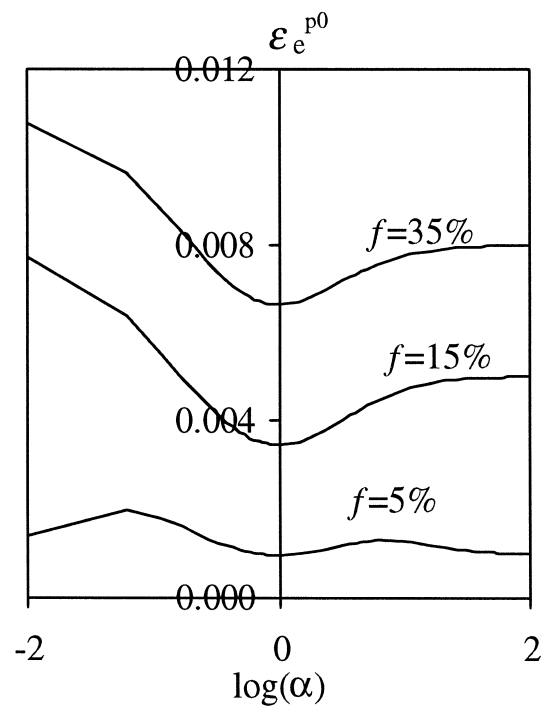

Fig. 1. The induced effective plastic strain in the matrix at the end of cooling as a function the aspect ratio of inclusions $\left(\Delta T=-500^{\circ} \mathrm{C}\right)$.

$h=577 \mathrm{MPa}, n=0.335$ and $\alpha_{0}=21.5 \times 10^{-6} /{ }^{\circ} \mathrm{C}$. For the inclusion $E_{1}=480 \mathrm{GPa}, \nu_{1}=0.17$ and $\alpha_{1}=3.8$ $\times 10^{-6} /{ }^{\circ} \mathrm{C}$.

Fig. 1 shows the equivalent plastic strain $\varepsilon_{\mathrm{e}}^{\mathrm{p} 0}$ at the end of a temperature drop $\Delta T=-500^{\circ} \mathrm{C}$ as a function of inclusion aspect ratio $(\alpha)$. It is seen that $\varepsilon_{\mathrm{e}}^{\mathrm{p} 0}$ increases with the volume fraction of the inclusions. At moderate concentration, the disk shape inclusions induce most pronounced effective plastic strain in the matrix and it is reversed for the spherical inclusion. For a temperature drop $\Delta T=-50^{\circ} \mathrm{C}$, during which no plastic flow is generated in the matrix, Fig. 2 illustrates the predicted difference in the initial yield stresses of the composite in tension and in compression $\left(\Sigma_{\mathrm{c}} / \Sigma_{\mathrm{t}}-1\right)$ as a function of inclusion aspect ratio. In Fig. $2, \Sigma_{\mathrm{c}}$ and $\Sigma_{\mathrm{t}}$ are the absolute yield stresses of the composite in compression and tension. For composites reinforced by prolate inclusions $(\alpha>1)$ the difference increases significantly as the aspect ratio of the inclusions increase and then

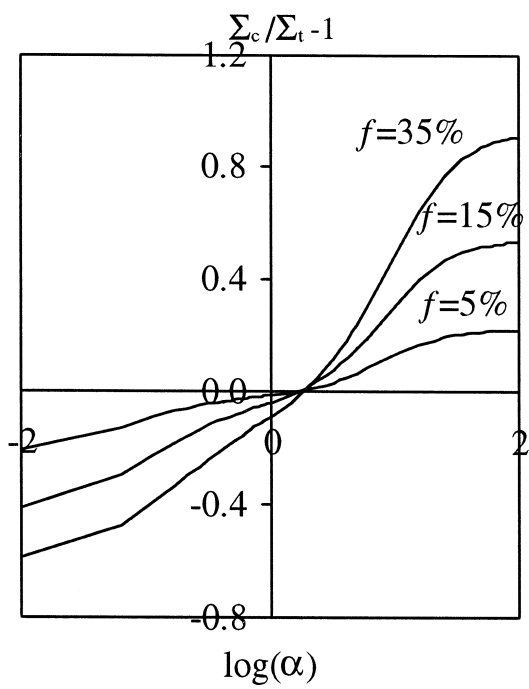

Fig. 2. The difference in the compressive and tensile yield stresses of the composite $\left(\Delta T=-50^{\circ} \mathrm{C}\right)$. 


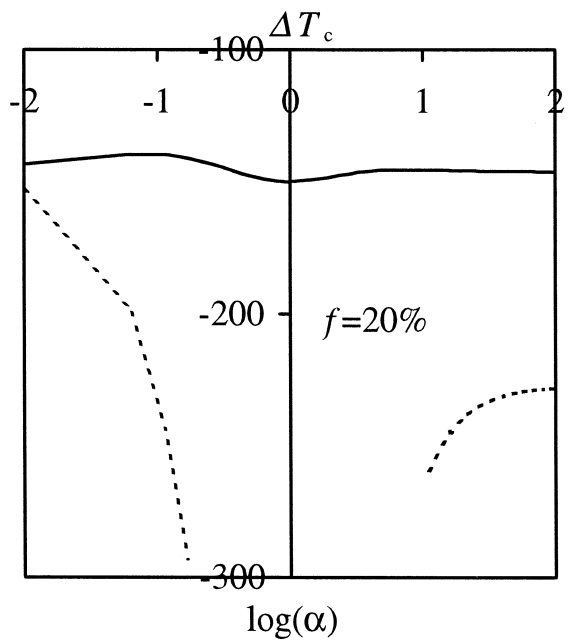

Fig. 3. The predicted temperature drop to generate the first plastic flow in the matrix as a function of inclusion aspect ratio (dashed lines: mean stress method).

stabilizes for long fiber like inclusions $(\alpha>100)$. For particulate-reinforced composites $(\alpha=1)$, the predicted initial yield stress in tension is greater than that in compression.

This has been confirmed by experimental observations (Corbin et al., 1991; Corbin and Wilkinson, 1994a,b). The difference in tensile and compressive hardening curves of the composite increases as a function of the volume fraction of inclusion. Fig. 3 shows the needed temperature drop $\left(\Delta T_{\mathrm{c}}\right)$ to generate first plastic flow in the matrix predicted by the mean stress approach and by the proposed method as a function of the inclusion's aspect ratio. The predicted temperature drop by the mean stress method is always higher than that predicted by the present method, especially for particulate reinforced composites, since the mean stress approach predicts no plastic flow. The influence of thermal residual stress on the subsequent plastic flow of their composite is given in Fig. 4 for different inclusion aspect ratios with a temperature drop $\Delta T=-500^{\circ} \mathrm{C}$. For problate inclusions

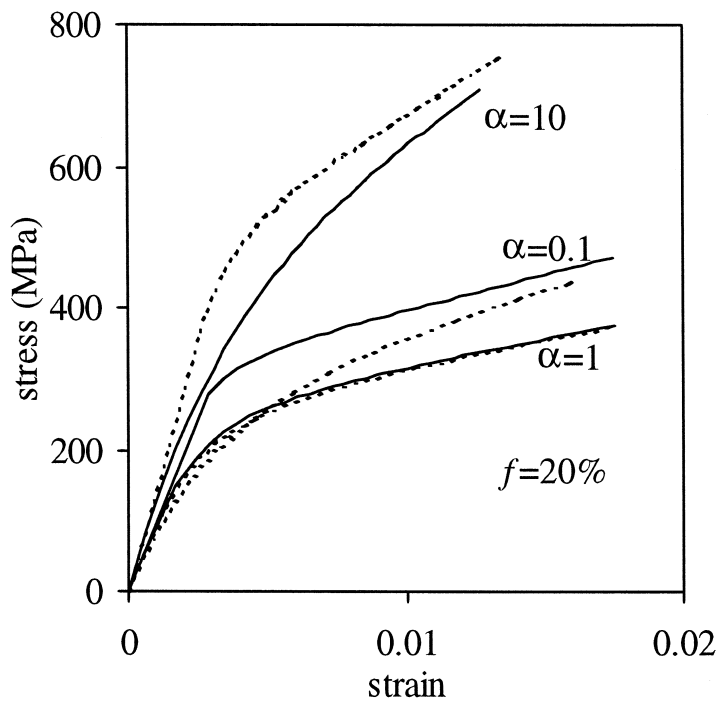

Fig. 4. Influence of residual stress on the stress-strain curves in tension (solid lines) and compression (dashed lines) of a nonlinear composite $\left(\Delta T=-500^{\circ} \mathrm{C}\right)$. 

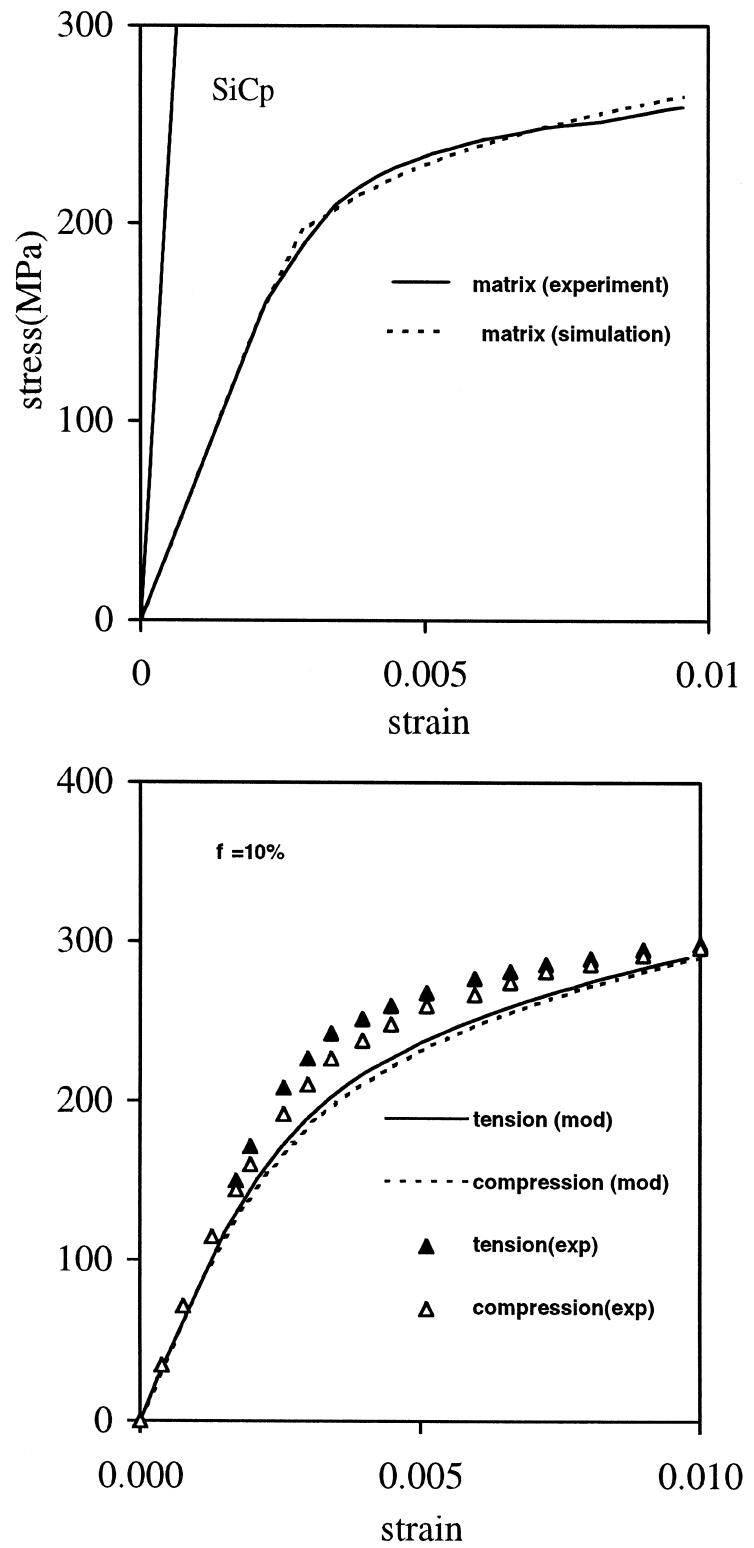

Fig. 5. (a) Stress-strain curve of the inclusions and the matrix material. (b) Influence of residual stress on the stress-strain curves in tension (solid lines) and compression (dashed lines) of a particulate composite together with experimental results (Corbin and Wilkinson, 1994a) $\left(\Delta T=-500^{\circ} \mathrm{C}\right)$.

with an aspect ratio larger than about 2 (see Fig. 2 for $\alpha=10$ ), the predicted hardening curve in compression is superior to that in tension and the results are reversed with oblate inclusions $(\alpha=0.1)$. In all cases the difference in tensile and compressive hardening curves of the composite decreases rapidly as a function of deformation. Due to the presence of internal thermal stresses in each phase, subsequent mechanical loading can immediately induce plastic flow in the matrix. The predicted initial yield stress of the composite in compression with problate inclusions and in tension for oblate inclusions are quite low (even zero). 
Finally the theory will be compared with the experimental observations of Corbin and Wilkinson (1994a,b) for a composite with $f=10 \%$. To provide a reference the stress-strain curves of the $\mathrm{SiC}$ inclusions and $\mathrm{Al}$ matrix are shown as Fig. 5(a) and the tensile and compressive hardening curves of the composite are depicted in Fig. 5(b). Both the theoretical calculations and experimental data in Fig. 5(b) indicate a higher tensile curve than the compressive one. The predicted difference in tensile and compressive hardening curves increases as a function of inclusion concentration. This difference is smoothed out quickly as plastic strain increases, and this agrees with the experimental tendency. The model, however, underestimates the hardening curves both in tension and compression. This may be attributed to the nonhomogeneous distribution of the inclusions (cluster) and to the irregular inclusion shape, which are not taken into account in the model. The presence of the cluster of inclusions can enhance the hardening curve of the composite (Corbin and Wilkinson, 1994a,b) and the irregular shape of the inclusions will certainly influence the local hardening. These effects are under investigation.

\section{Summary}

The thermal residual stresses generated during a temperature variation and their influence on the subsequent mechanical behaviors of composites reinforced with aligned inclusions are analyzed through a new method. This method is based on a secant moduli approximation and the homogenized effective stress of the matrix, which was proposed by Qiu and Weng (1992) and evaluated exactly in this paper in the presence of thermal residual stresses. The analytical results show that the induced plastic strain (after a temperature change) and their influence on the mechanical behavior depends significantly on the inclusion shape. With prolate inclusions, the presence of thermal residual stresses generate a higher compressive hardening curve of the composite, but it is reversed with oblate inclusions. For particle reinforced composite $(\alpha=1)$, thermal residual stresses induce a tensile hardening curve higher than the compressive one and this is in agreement with experimental observations. In all cases the induced asymmetry in tensile and compressive hardening curves is smoothed out quickly with the plastic deformation of the ductile matrix.

\section{Appendix A}

Consider a representative volume element (RVE), with aligned spheroidal inclusions homogeneously dispersed in the matrix. A macroscopic stress $\boldsymbol{\Sigma}$ (or strain $\boldsymbol{E}$ ) is applied along the boundary of RVE. In each inclusion, there is a uniform eigenstrain $\boldsymbol{\varepsilon}^{*}$. Both phases are assumed to be elastic. Taking the matrix as the comparison composite, $\boldsymbol{E}_{0}$ is defined by $\boldsymbol{\Sigma}=\boldsymbol{L}_{0} \boldsymbol{E}_{0}$, and the matrix average stress in the composite is given by $\boldsymbol{\sigma}_{0}=\boldsymbol{L}_{0}\left(\boldsymbol{E}_{0}+\boldsymbol{\varepsilon}_{\mathrm{p} 0}\right)$ and the average strain in the matrix is $\boldsymbol{\varepsilon}_{0}=\boldsymbol{E}_{0}+\boldsymbol{\varepsilon}_{\mathrm{p} 0}$. The average strain in the inclusions differs further from the matrix average strain by $\boldsymbol{\varepsilon}_{\mathrm{p} 1}$, so that the average stress of the inclusions is

$\boldsymbol{\sigma}_{1}=\boldsymbol{L}_{1}\left(\boldsymbol{E}_{0}+\boldsymbol{\varepsilon}_{\mathrm{p} 0}+\boldsymbol{\varepsilon}_{\mathrm{p} 1}-\boldsymbol{\varepsilon}^{*}\right)=\boldsymbol{L}_{0}\left(\boldsymbol{E}_{0}+\boldsymbol{\varepsilon}_{\mathrm{p} 0}+\boldsymbol{\varepsilon}_{\mathrm{p} 1}-\boldsymbol{\varepsilon}^{*}-\boldsymbol{\varepsilon}^{\mathrm{T}}\right)$,

where $\boldsymbol{\varepsilon}^{\mathrm{T}}$ is the eigenstrain introduced by Eshelby (1957) and

$\boldsymbol{\varepsilon}_{\mathrm{p} 1}=\boldsymbol{S}\left(\boldsymbol{\varepsilon}^{*}+\boldsymbol{\varepsilon}^{\mathrm{T}}\right)$

$\boldsymbol{S}$ being the Eshelby tensor. Using the fact $\langle\boldsymbol{\sigma}\rangle=(1-f)\langle\boldsymbol{\sigma}\rangle_{0}+f\langle\boldsymbol{\sigma}\rangle_{1}=\boldsymbol{\Sigma}$, we get

$\boldsymbol{\varepsilon}_{\mathrm{p} 0}+f\left(\boldsymbol{\varepsilon}_{\mathrm{p} 1}-\boldsymbol{\varepsilon}^{*}-\boldsymbol{\varepsilon}^{\mathrm{T}}\right)=0$. 
From Eqs. (A.1), (A.2) and (A.3), the unknown quantities $\boldsymbol{\varepsilon}_{\mathrm{p} 1}, \boldsymbol{\varepsilon}_{\mathrm{p} 0}, \boldsymbol{\varepsilon}^{\mathrm{T}}$ can be evaluated and the composite strain is calculated by

$\boldsymbol{E}=(1-f) \boldsymbol{\varepsilon}_{0}+f \boldsymbol{\varepsilon}_{1}=\boldsymbol{E}_{0}+f\left(\boldsymbol{\varepsilon}^{*}+\boldsymbol{\varepsilon}^{\mathrm{T}}\right)$,

where

$$
\begin{aligned}
\boldsymbol{\varepsilon}^{\mathrm{T}}= & \left\{\left[\boldsymbol{M}_{1} \boldsymbol{M}_{0}^{-1}-\boldsymbol{I}\right]^{-1}+(1-f)(\boldsymbol{I}-\boldsymbol{S})\right\}^{-1} \boldsymbol{E}_{0}-(1-f)\left\{\left[\boldsymbol{M}_{1} \boldsymbol{M}_{0}^{-1}-\boldsymbol{I}\right]^{-1}\right. \\
& +(1-f)(\boldsymbol{I}-\boldsymbol{S})\}^{-1}(\boldsymbol{I}-\boldsymbol{S}) \boldsymbol{\varepsilon}^{*}
\end{aligned}
$$

and the composite compliance tensor has the form

$\boldsymbol{M}_{\mathrm{c}}=\boldsymbol{M}_{0}+f\left\{\left[\boldsymbol{M}_{1} \boldsymbol{M}_{0}^{-1}-\boldsymbol{I}\right]^{-1}+(1-f)(\boldsymbol{I}-\boldsymbol{S})\right\}^{-1} \boldsymbol{M}_{0}$.

The average stresses of the matrix and inclusions can be found as:

$$
\begin{aligned}
& \langle\boldsymbol{\sigma}\rangle_{0}=\frac{1}{1-f}\left(\boldsymbol{M}_{1}-\boldsymbol{M}_{0}\right)^{-1}\left(\boldsymbol{M}_{1}-\boldsymbol{M}_{\mathrm{c}}\right) \boldsymbol{\Sigma}-\left\{\left(\boldsymbol{M}_{1}-\boldsymbol{M}_{0}\right)^{-1}\left(\boldsymbol{M}_{1}-\boldsymbol{M}_{\mathrm{c}}\right)-\boldsymbol{I}\right\} \boldsymbol{M}_{0}^{-1}(\boldsymbol{I}-\boldsymbol{S}) \boldsymbol{\varepsilon}^{*}, \\
& \langle\boldsymbol{\sigma}\rangle_{1}=\frac{1}{1-f}\left(\boldsymbol{M}_{1}-\boldsymbol{M}_{0}\right)^{-1}\left(\boldsymbol{M}_{\mathrm{c}}-\boldsymbol{M}_{0}\right) \boldsymbol{\Sigma}-\frac{1-f}{f}\left(\boldsymbol{M}_{1}-\boldsymbol{M}_{0}\right)^{-1}\left(\boldsymbol{M}_{\mathrm{c}}-\boldsymbol{M}_{0}\right) \boldsymbol{M}_{0}^{-1}(\boldsymbol{I}-\boldsymbol{S}) \boldsymbol{\varepsilon}^{*}
\end{aligned}
$$

or separately in terms of the applied stress $\boldsymbol{\Sigma}$ and the eigenstrain $\boldsymbol{\varepsilon}^{*}$

$$
\begin{aligned}
\left\langle\boldsymbol{\sigma}^{\mathrm{I}}\right\rangle_{0} & =\frac{1}{1-f}\left(\boldsymbol{M}_{1}-\boldsymbol{M}_{0}\right)^{-1}\left(\boldsymbol{M}_{1}-\boldsymbol{M}_{\mathrm{c}}\right) \boldsymbol{\Sigma}, \\
\left\langle\boldsymbol{\sigma}^{\mathrm{II}}\right\rangle_{0} & =-\left\{\left(\boldsymbol{M}_{1}-\boldsymbol{M}_{0}\right)^{-1}\left(\boldsymbol{M}_{1}-\boldsymbol{M}_{\mathrm{c}}\right)-\boldsymbol{I}\right\} \boldsymbol{M}_{0}^{-1}(\boldsymbol{I}-\boldsymbol{S}) \boldsymbol{\varepsilon}^{*}, \\
\left\langle\boldsymbol{\sigma}^{\mathrm{I}}\right\rangle_{1} & =\frac{1}{f}\left(\boldsymbol{M}_{1}-\boldsymbol{M}_{0}\right)^{-1}\left(\boldsymbol{M}_{\mathrm{c}}-\boldsymbol{M}_{0}\right) \boldsymbol{\Sigma}, \\
\left\langle\boldsymbol{\sigma}^{I I}\right\rangle_{1} & =\frac{1-f}{f}\left(\boldsymbol{M}_{1}-\boldsymbol{M}_{0}\right)^{-1}\left(\boldsymbol{M}_{c}-\boldsymbol{M}_{0}\right) \boldsymbol{M}_{0}^{-1}(\boldsymbol{I}-\boldsymbol{S}) \boldsymbol{\varepsilon}^{*} .
\end{aligned}
$$

For a particulate composite $(\alpha=1)$, the composite as a whole is isotropic and the stress (or strain) can be decomposed as $\sigma_{i j}=s_{i j}+1 / 3 \sigma \delta_{i j}$ (for a strain $\varepsilon_{i j}=e_{i j}+1 / 3 \theta \delta_{i j}$ ). The elastic constitutive relationship for an isotropic material reads

$s_{i j}=2 \mu e_{i j}, \quad \sigma=3 k \theta$.

The Eshelby relationship (Eq. (A.2)) for a particle-reinforced composite can be written as $e_{i j}^{\mathrm{p} 1}=\beta\left(e_{i j}^{*}+e_{\mathrm{ij}}^{\mathrm{T}}\right)$, $\theta^{\mathrm{p} 1}=\alpha\left(\theta^{*}+\theta^{\mathrm{T}}\right)$, where $\alpha=\left(1+\nu_{0}\right) / 3\left(1-\nu_{0}\right), \beta=2\left(4-5 \nu_{0}\right) / 15\left(1-\nu_{0}\right)$ and for a macroscopic stress $\Sigma_{i j}=S_{i j}+1 / 3 \operatorname{tr} \mathbf{\Sigma} \delta_{i j}$.

By rewriting Eqs. (A.1), (A.2) and (A.3) into the deviatoric and spherical parts and following the same procedure, we can get:

$$
\frac{k_{\mathrm{c}}}{k_{0}}=1+\frac{f\left(k_{1}-k_{0}\right)}{(1-f) \alpha\left(k_{1}-k_{0}\right)+k_{0}}, \quad \frac{\mu_{\mathrm{c}}}{\mu_{0}}=1+\frac{f\left(\mu_{1}-\mu_{0}\right)}{(1-f) \beta\left(\mu_{1}-\mu_{0}\right)+\mu_{0}} .
$$


The average stresses in the inclusions are

$$
\begin{array}{ll}
\left\langle\sigma^{\mathrm{I}}\right\rangle_{1}=\frac{k_{1}}{(1-f)(\alpha-1)\left(k_{1}-k_{0}\right)+k_{1}} \operatorname{tr} \Sigma, & \left\langle\sigma^{\mathrm{II}}\right\rangle_{1}=\frac{3 k_{0} k_{1}(1-f)(\alpha-1)}{(1-f)(\alpha-1)\left(k_{1}-k_{0}\right)+k_{1}} \theta^{*}, \\
\left\langle s_{i j}^{\mathrm{I}}\right\rangle_{1}=\frac{\mu_{1}}{(1-f)(\beta-1)\left(\mu_{1}-\mu_{0}\right)+\mu_{1}} S_{i j}, & \left\langle s_{i j}^{\mathrm{II}}\right\rangle_{1}=\frac{3 \mu_{0} \mu_{1}(1-f)(\beta-1)}{(1-f)(\beta-1)\left(\mu_{1}-\mu_{0}\right)+\mu_{1}} e_{i j}^{*},
\end{array}
$$

whereas the average stresses in the matrix are

$$
\begin{array}{ll}
\left\langle\sigma^{\mathrm{I}}\right\rangle_{0}=\frac{\alpha\left(k_{1}-k_{0}\right)+k_{0}}{(1-f)(\alpha-1)\left(k_{1}-k_{0}\right)+k_{1}} \operatorname{tr} \Sigma, & \left\langle\sigma^{\mathrm{II}}\right\rangle_{0}=-\frac{3 k_{0} k_{1} f(\alpha-1)}{(1-f)(\alpha-1)\left(k_{1}-k_{0}\right)+k_{1}} \theta^{*}, \\
\left\langle s_{i j}^{\mathrm{I}}\right\rangle_{0}=\frac{\beta\left(\mu_{1}-\mu_{0}\right)+\mu_{0}}{(1-f)(\beta-1)\left(\mu_{1}-\mu_{0}\right)+\mu_{1}} S_{i j}, & \left\langle s_{i j}^{\mathrm{II}}\right\rangle_{0}=-\frac{3 \mu_{0} \mu_{1} f(\beta-1)}{(1-f)(\beta-1)\left(\mu_{1}-\mu_{0}\right)+\mu_{1}} e_{i j}^{*} .
\end{array}
$$

\section{References}

Arsenault, R.J., Taya, M., 1987. Thermal residual stress in metal matrix composite. Acta metall. mater. 35, 651-659.

Berveiller, M., Zaoui, A., 1979. An extension of the self-consistent scheme to plastically flowing polycrystals. J. Mech. Phys. Solids. 26, 325-344.

Bhattacharyya, A., Sakaki, T., Weng, G.J., 1993. The influence of Martensite shape, concentration and phase transformation strain on the deformation behavior of stable dual-phase steels. Metall. Trans. 24A, 301-314.

Bobeth, M., Diener, G., 1987. Static elastic and thermoelastic field fluctuation in multiphase composites. J. Mech. Phys. Solids. 35, 137-149.

Corbin, S.F., Wilkinson, D.S., 1994a. Influence of matrix strength and damage accumulation on the mechanical response of a particulate metal matrix composite. Acta metall. mater. 42, 1319-1328.

Corbin, S.F., Wilkinson, D.S., 1994b. The influence of particle distribution on the mechanical response of a particulate metal matrix composite. Acta metall. mater. 42, 1311-1318.

Corbin, S.F., Wilkinson, D.S., Embury, J.D., 1991. The onset of yielding in a SiCp reinforced A1 alloy. Proceeding of 12th Riso International Symposium on Materials Science. pp. 283-290.

Corbin, S.F., Wilkinson, D.S., Embury, J.D., 1996. The Bauschinger effect in a particle reinforced Al alloy. Mater. Sci. Eng. A $207,1-11$.

Eshelby, J.D., 1957. The determination of the elastic field of an ellipsoidal inclusion and related problems. Proc. R. Soc. London Ser. A: 241, 376-396.

Hill, R., 1963. Elastic properties of reinforced solids: Some theoretical principles. J. Mech. Phys. Solids. 11, 357-372.

Hu, G.K., 1996. A method of plasticity for general aligned spheroidal void or fiber reinforced composites. Int. J. Plast. 12, 439-449.

Kreher, W., 1990. Residual stress and stored elastic energy of composites and polycrystals. J. Mech. Phys. Solids. 38, $115-128$.

Mori, T., Tanaka, K., 1973. Average stress in the matrix and average elastic energy of materials with misfitting inclusions. Acta metall. mater. 21, 571-574.

Pan, H.H., Weng, G.J., 1993. Thermal stress relief by plastic deformation in aligned two-phase composites. Compos. Eng. 3, $219-234$.

Ponte Castaneda, P., 1991. The effective mechanical properties of nonlinear isotropic composites. J. Mech. Phys. Solids. 39, 45-71.

Qiu, Y.P., Weng, G.J., 1992. A theory of plasticity for porous materials and particle-reinforced composites. ASME J. Appl. Mech. 59, 261-268.

Ramakrishnan, N., 1996. An analytical study on strengthening of particulate reinforced metal matrix composites. Acta metall. 44, 69-77.

Shibata, S., Taya, M., Mori, T., Mura, T., 1992. Dislocation punching from spherical inclusions in a metal matrix composites. Acta metall. mater. 40, 3141-3148.

Takao, Y., Taya, M., 1985. Thermal expansion coefficients and thermal stresses in the aligned short fiber composite with application to a short carbon fiber/aluminium. ASME J. Appl. Mech. 52, 806-810. 
Tandon, G.P., Weng, G.J., 1988. A theory of particle-reinforced plasticity. ASME J. Appl. Mech. 55, $126-135$.

Taya, M., Mori, T., 1987. Dislocation punched-out around a short fiber in a short fiber matrix composite subjected to uniform temperature change. Acta metall. mater. 35, 155-162.

Withers, P.J., Stobbs, W.M., Pedersen, O.B., 1989. The application of the Eshelby method of internal stress determination for short fiber metal matrix composites. Acta metall. mater. 37, 2084-3061. 